\title{
CHANDRA CHARACTERIZATION OF X-RAY EMISSION IN THE YOUNG F-STAR BINARY SYSTEM HD 113766
}

\author{
C. M. $\operatorname{Lisse}^{1}$, D. J. Christian ${ }^{2}$, S. J. Wolk ${ }^{3}$, H. M. GÜnther ${ }^{4}$, C. H. Chen ${ }^{5}$, and C. A. Grady ${ }^{6}$ \\ ${ }^{1}$ Planetary Exploration Branch, Space Exploration Sector, Johns Hopkins University Applied Physics Laboratory, \\ 11100 Johns Hopkins Road, Laurel, MD 20723, USA; carey.lisse@jhuapl.edu \\ 2 Department of Physics and Astronomy, California State University Northridge, 18111 Nordhoff Street, \\ Northridge, CA 91330, USA; damian.christian@csun.edu \\ ${ }^{3}$ Chandra X-ray Center, Harvard-Smithsonian Center for Astrophysics, 60 Garden Street, \\ Cambridge, MA 02138, USA; swolk@cfa.harvard.edu \\ ${ }^{4}$ Massachusetts Institute of Technology, Kavli Institute for Astrophysics and Space Research, \\ 77 Massachusetts Avenue, NE83-569, Cambridge, MA 02139, USA; hgunther@mit.edu \\ ${ }^{5}$ STScI, 3700 San Martin Drive, Baltimore, MD 21218, USA; cchen@stsci.edu \\ ${ }^{6}$ Eureka Scientific and Goddard Space Flight Center, Code 667, NASA-GSFC, Greenbelt, MD 20771, USA; carol.a.grady@nasa.gov \\ Received 2016 January 14; revised 2016 September 8; accepted 2016 September 23; published 2017 January 10
}

\begin{abstract}
Using Chandra, we have obtained imaging X-ray spectroscopy of the 10-16 Myr old F-star binary HD 113766. We individually resolve the 1".4 separation binary components for the first time in the X-ray and find a total $0.3-2.0 \mathrm{keV}$ luminosity of $2.2 \times 10^{29} \mathrm{erg} \mathrm{s}^{-1}$, consistent with previous RASS estimates. We find emission from the easternmost, infrared-bright, dusty member HD 113766A to be only $10 \%$ that of the western, infrared-faint member HD 113766B. There is no evidence for a 3rd late-type stellar or substellar member of HD 113766 with $L_{\mathrm{x}}>6 \times 10^{25} \mathrm{erg} \mathrm{s}^{-1}$ within $2^{\prime}$ of the binary pair. The ratio of the two stars' X-ray luminosity is consistent with their assignments as F2V and F6V by Pecaut et al. The emission is soft for both stars, $k T_{\text {Apec }}=0.30-0.50 \mathrm{keV}$, suggesting X-rays produced by stellar rotation and/or convection in young dynamos, but not accretion or outflow shocks, which we rule out. A possible $2.8 \pm 0.15(2 \sigma) \mathrm{hr}$ modulation in the HD 113766B X-ray emission is seen, but at very low confidence and of unknown provenance. Stellar wind drag models corresponding to $L_{\mathrm{x}} \sim 2 \times 10^{29} \mathrm{erg} \mathrm{s}^{-1}$ argue for a $1 \mathrm{~mm}$ dust particle lifetime around HD $113766 \mathrm{~B}$ of only $\sim 90,0000$ years, suggesting that dust around HD 113766B is quickly removed, whereas $1 \mathrm{~mm}$ sized dust around HD 113766A can survive for $>1.5 \times 10^{6}$ years. At $10^{28}-10^{29} \mathrm{erg} \mathrm{s}^{-1} \mathrm{X}$-ray luminosity, astrobiologically important effects, like dust warming and X-ray photolytic organic synthesis, are likely for any circumstellar material in the HD 113766 systems.
\end{abstract}

Key words: astrochemistry - protoplanetary disks - planets and satellites: formation - techniques: spectroscopic X-rays: stars

\section{INTRODUCTION}

We report here on an analysis of Chandra soft X-ray observations of HD 113766, a young (10-16 Myr old, Mamajek et al. 2002; Chen et al. 2006, 2011; Lisse et al. 2008; Pecaut et al. 2012), F-star binary stellar system of near-solar metallicity $(\mathrm{Fe} / \mathrm{H}=-0.1, \quad$ Nordstrom et al. 2004), located at a distance of $123^{+18} /-14$ pc $(8.16$ mas Hipparcos parallax) from the Earth (Van Leeuwen 2007). Little is known about this system in the X-ray, other than it is a reported unresolved RASS source of luminosity 2.1 $+/-0.7 \times 10^{29} \mathrm{erg} \mathrm{s}^{-1}$. On the other hand, in the optical/IR, the system is very interesting. With two component stars of nearly identical age characterized by $\mathrm{F}$ spectral types in the Sco-Cen star-forming association, attention had been called to this system, since its association with object IRAS 13037-4545 in the IRAS Point Source Catalog was found (Backman \& Paresce 1993). More recent work by Meyer et al. (2001), Lisse et al. (2008), and Chen et al. (2005, 2006, 2011) have confirmed that the system exhibits unobscured photospheres and that HD 113766A exhibits one of the largest IR flux excesses measured $\left(L_{\mathrm{IR}} / L_{*}=0.015\right)$, with no detectable $\mathrm{H}_{2}$ emission. HD 113766A thus belongs to the class of post-T Tauri objects characterized by young ages of 5-30 Myr, no leftover primordial gas, and large quantities of excess mid-IR emission from circumstellar dust. On the other hand, there is no evidence for circumstellar dust orbiting the companion, coeval F-star HD 113766B (Meyer et al. 2001), even though studies of young stellar clusters (e.g., $h$ and $\chi$ Persei, Currie et al. 2007, 2008) would have led us to expect a few similar dust-forming collisions every Myr in HD 113766B.

Late F-stars are typically strong X-ray emitters at a young age. In fact, the X-ray luminosity function of the Hyades open cluster (age $600 \mathrm{Myr}$ ) peaks at late F-stars (Stern et al. 1995). While X-ray emission of $\mathrm{O}$ to mid B-type stars is attributed to dissipating shocks in radiation driven winds, and low-mass GKM stars have strong convection leading to an $\alpha \omega$ or $\alpha^{2}$ dynamo, the origin of emission in late B to early F-stars is not so clear. While such stars have a convection zone, the ratio of the X-ray to bolometric flux is much smaller than in typical PMS stars. For example, Collins et al. (2009) find the $10 \mathrm{Myr}$ debris disk host HD 100453 (A9Ve) to have log $L_{\mathrm{X}} / L_{\mathrm{bol}} \sim-5.9$, whereas the typical value for PMS GKM stars is about -3.5 (Feigelson et al. 2005). Intermediate mass stars also appear to have softer spectra than their lower-mass brethren. For example, HD 100453 and the similarly aged 51 Eri (F0V) have coronal temperatures of about $0.2 \mathrm{keV}$, as opposed to $1-2 \mathrm{keV}$ for similarly aged GKM stars (Feigelson et al. 2006; Collins et al. 2009). Finally, the "FIP-effect" in which elements with first ionization potential below about $10 \mathrm{eV}$ are observed to be enhanced in abundance by a factor of about 3 in the solar corona (Draker et al. 1995), appears to be 
absent in some F-stars such as $\tau$ Boo A (an F7V; Maggio et al. 2011), and Procyon (an F5IV; Raassen et al. 2002 - but see Wood \& Laming 2013 for a counter example).

The twin F-stars in the HD 113766 system are thus an interesting and useful couple to study. Close enough (separated by only 1 !" 4 , or $170 \mathrm{AU}$ ) to be in the same interstellar medium (ISM) environment, far enough separated that they minimally influence each other's circumstellar environment inside $100 \mathrm{au}$, and formed at the same time with about the same total mass, they are a natural testbed for trying to understand the mechanisms of exosystem formation. As Myr-old F-stars can be expected to be fast rotators, and more convective and X-ray active than their main-sequence cousins, we would have naively expected both stars to be rapidly rotating, highly convective, and X-ray bright. We thus obtained Chandra observations of HD 113766 in 2010, because: (a) the system contained a well known and well studied IRAS and WISE debris disk, while also being a known RASS source, and therefore observable by Chandra, which is a rare combination; (b) using Chandra, we could produce the very first resolved maps for the 1". 4 wide binary; and (c) it was important to measure the first resolved X-ray spectrum of the system in order to characterize the stellar wind environment of each of its stars, and understand their effect on the known massive dust and ice belts around HD 113766A.

\section{OBSERVATIONS}

In this section, we present the circumstances and results of our 2010 Chandra observations of the HD 113766 system. In the next Section 3, we will discuss their implications.

\subsection{CXO Photometry and Luminosity}

We observed the HD 113766 binary using Chandra ACIS-S imaging spectroscopy under Chandra X-ray Observatory $(C X O)$ program OBSID 12384 on 2010 December 02 UT. For the observation, no filters or gratings were used, and the stars were centered in the 'sweet spot' of the S3 chip. We reprocessed the Chandra data with CIAO version 4.8 (Fruscione et al. 2006), which applied the energy-dependent subpixel event repositioning. The total program observing time was $39 \mathrm{ks}$, and the total on-target observing time was $37.3 \mathrm{ks}$, in which 1509 total raw photons were detected using a source extraction radius of $20^{\prime \prime}$ and an energy filter in the range $0.3-2.0 \mathrm{keV}$. The extraction region was centered halfway between the two resolved sources' centers. The background was estimated from a large, source-free region on the same chip to be 0.0120 counts per pixel ${ }^{-1}$ in $37.2 \mathrm{ks}\left(=3.2 \times 10^{-7} \mathrm{cps} /\right.$ pixel $)$.

After subtracting the background, we determined a total of 1370 source counts, and a source count rate of 0.0366 cps. Using the RASS PSPC rate of $0.034 \pm 0.014 \mathrm{cps}$ (ROSAT AllSky Survey, Voges et al. 1999) and assuming a RaymondSmith coronal plasma model with $\log T=6.2$ and solar abundances (Raymond \& Smith 1977), W3PIMMS indicated a total HD 113766A $+\mathrm{B}$ effective Chandra count rate of 0.032 cps (assuming a $1 / 4$ subarray to address pileup). Thus, the observed average Chandra count rate was within $10 \%$ of the RASS count rate extrapolated to the ACIS-S (assuming coronal emission).

The photometric time series (light curve) of the Chandra observations is shown for the two sources in Figure 1. The 10:1 relative level of brightness of the two sources remains stable over the $10.5 \mathrm{hr}$ of Chandra observation to within the statistical photon noise. There are qualitatively, however, potential variations in the HD $113766 \mathrm{~B}$ light curve, which could be periodic. Analyzing the data for possible sinusoidal variations, we find a number of possible solutions with periods ranging from $2.61-2.92 \mathrm{hr}$, with a best-fit solution at $2.87 \mathrm{hr}$, a peak-topeak amplitude of $20 \%$, and $\chi^{2}{ }_{\nu}=0.98$ for 36 degrees of freedom (dof). However, we also find a null periodic solution with $\chi_{\nu}^{2}=1.23$, and note that the $95 \%$ confidence limit of the $\chi_{\nu}^{2}$ distribution for 36 dof is 1.42 .

While it is tempting to assign this periodicity to rotationally induced variability, the equatorial velocity implied for a 1.35 $R_{\text {Sun }}$ F6V star is $423 \mathrm{~km} \mathrm{~s}^{-1}$, higher than the predicted breakup speed for a solar abundance F-star (for $M_{*}=1.2-1.6 M_{\text {Sun }}$, $v_{\text {breakup }} \sim 300 \mathrm{~km} \mathrm{~s}^{-1}$ ). The implied equatorial velocity is also approximately a factor of 2 higher than the fastest known stellar rotators (Głebocki \& Gnaciński 2003; Chen et al. 2011). On the other hand, Chen et al. (2011) have listed a value of $v \sin i=93 \mathrm{~km} \mathrm{~s}^{-1}$ for HD 113766B, and Smith et al. (2012) and Olafsson et al. (2013) have published models of the HD 113766A disk with inclination $i<10^{\circ}$; if HD 113766B has a similar inclination, it could have an equatorial rotation velocity in the order of $400 \mathrm{~km} \mathrm{~s}^{-1}$, consistent with the observations. We thus note the possible X-ray periodicity in our HD 113766B data for future reference, but also note that we do not understand its source if it is real. It will take a deeper, longer set of X-ray observations than is presented here to robustly verify this possibility.

\section{2. $C X O$ Imaging}

On the S3 chip, we detected two closely spaced X-ray sources in the raw HD 113766 imagery (Figure 2(a)). Image deconvolution was able to better separate these down to the 0 ". 2 scale, clearly showing two separate sources (Figure 2(b)). The centers of these lie $\sim 1$ ". 4 apart, and are entirely consistent with the optical and IR locations and separation of $\sim 1$ ". 4 for the two stars (Lisse et al. 2008 and references therein), with the optical and IR-brighter HD 113766A to the east, and the optical and IR-fainter but X-ray brighter HD $113766 \mathrm{~B}$ to the west. The observed flux is very asymmetrically distributed, with $\sim 90 \%$ arising from the optically fainter HD 113766B western binary member, and $\sim 10 \%$ arising from the optically brighter HD 113766 A eastern binary member.

In the $2^{\prime} \times 2^{\prime}$ subarray field around the HD 113766 binary pair, we performed a companion search for potential nearby $\mathrm{X}$-ray sources in the field. There is no evidence in our data for any separate, X-ray bright 3rd member of HD 113766 within the $2^{\prime}$ of the binary pair with flux greater than three times the image background level. Given the background level of $\sim 3.2 \times 10^{-7}$ cps per pixel, and an ACIS-S 90\% encircled energy radius of 5 pixels, this implies a $3 \sigma$ upper limit for any $\mathrm{X}$-ray object in the field of $7.5 \times 10^{-5} \mathrm{cps}$ (approximately equivalent to $L_{\mathrm{x}} \sim 5 \times 10^{26} \mathrm{erg} \mathrm{s}^{-1}$, assuming $k T=0.40 \mathrm{keV}$ ) for an object at the $130 \mathrm{pc}$ distance of HD $113766 \mathrm{~A} / \mathrm{B}$. This null result is consistent with the lack of any RASS sources in a $4 ! 2 \times 4 ! 2$ field centered on HD $113766 \mathrm{~A} / \mathrm{B}$ other than the binary system itself (Voges et al. 1999).

Any optically faint, coeval 10-16 Myr old KM stellar or L/T brown-dwarf class object should have been easily detected in the our ACIS-S imaging (see, for example, the strong Chandra detection of the M2.5V HR 4796B binary companion to the A0V HR 4796A disk system by Drake 

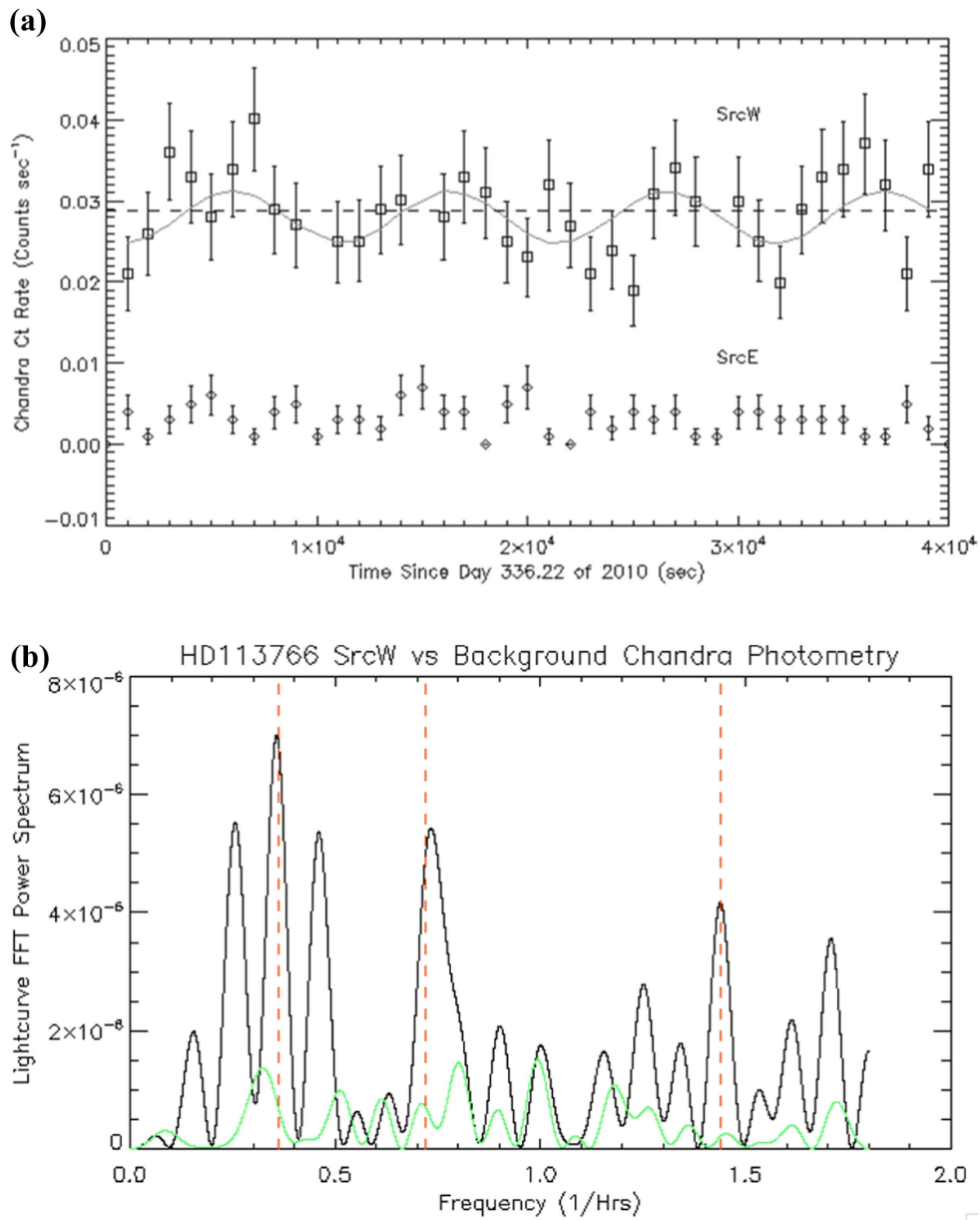

Figure 1. (a) Chandra ACIS-S photometry of the two X-ray sources detected in the HD 113766 system. All error bars and error estimates are $2 \sigma$. The background count rate in the Chandra point-spread function, as measured far off-source, has been measured with level $0.01+/-0.002 \mathrm{cps}$ and removed from these curves. The western source, labeled "SrcW," and identified with HD 113766B, demonstrates a flux of $0.029+/-0.005$ cps (squares), with a possible modulation with sinusoidal periodicity of $2.8( \pm 0.15) \mathrm{hr}$ and amplitude $0.0033( \pm 10 \%) \mathrm{cps}$ (gray curve) ("possible" since a constant flux model (dashed line) also has $\chi^{\nu 2}$ value within the $95 \%$ confidence limits for our 36 dof light curve). The eastern source, labeled as "SrcE" and identified with HD 113766A (diamonds), is roughly 10 times fainter at $0.0037 \pm 0.002 \mathrm{cps}$. The detection of SrcE is at too low a significance to determine any modulation in the A source. (b) Power spectrum of the HD 113766B Chandra light curve (black) compared to that of the background (green), with the location of the possible $2.8 \mathrm{hr}$ periodicity and its $n=2$ and $n=4$ harmonics marked by the red dashed lines.

et al. 2014). Furthermore, the X-ray spectra we report below for the two stars is much too soft to be produced by an X-ray active KM class stellar object (Collins et al. 2009). While the Chandra data cannot rule out very close-in, optically faint substellar companions within 0".5 of the HD 113766 stars, which may be X-ray active, no substantial brown-dwarf-like infrared (IR) excess above the stellar photospheres of either component A or B has been found (Lisse et al. 2008, Olafsson et al. 2013) and $R-V$ measurements of the stars have put upper limits of $<10 M_{\text {Jup }}$ on any close-in companion masses within $50 \mathrm{AU}$ of their primaries (F. Galland et al. 2010, private communication).

\subsection{CXO Spectroscopy}

From the 1370 detected events, we produced a total HD 113766A+B spectrum, extracted over both sources with an $r=20$ pixel circular aperture. The combined spectrum is shown in Figure 3(a), and is soft. We estimate a total HD 113766 A+B system luminosity of $L_{\mathrm{x}}=2.2 \times 10^{29} \mathrm{erg} \mathrm{s}^{-1}$. We also extracted separate spectra for the "west" and "east" sources, each over an $r=1.6$ pixel circular aperture (Figure 3(a)). After allowing for 30 counts from the brighter western source in the fainter eastern source's aperture, this provided a reasonable separation of the two 
(a)

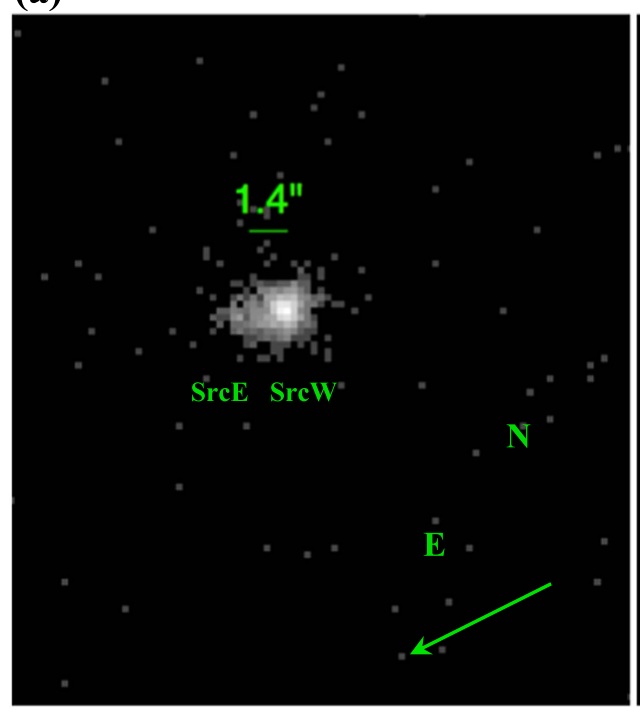

(b)

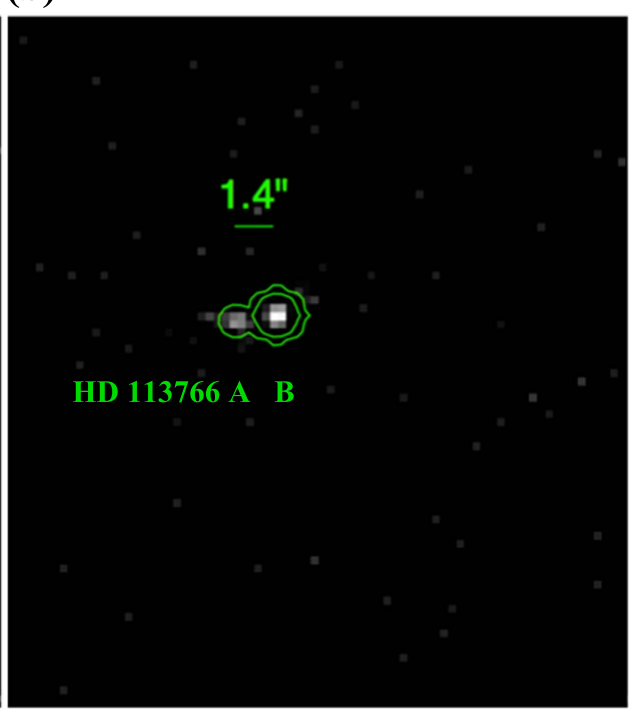

(c)

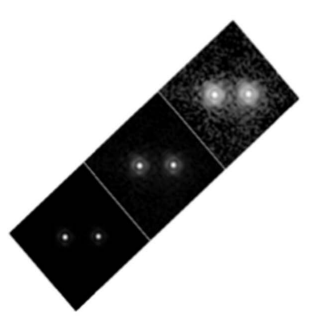

(d)

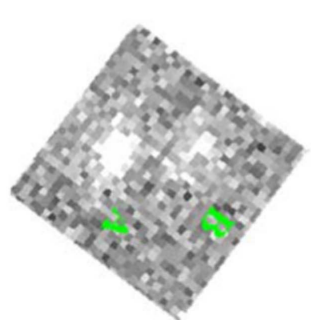

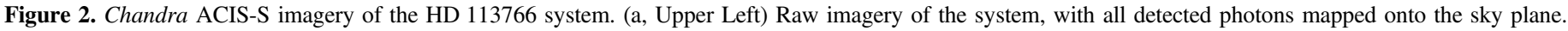

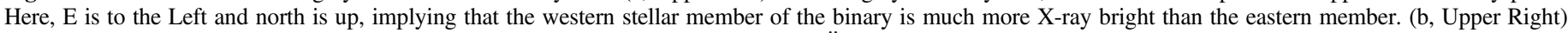

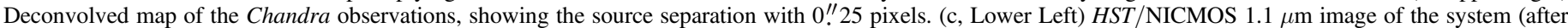
Meyer et al. 2001). (d, Lower Right) : Magellan $11 \mu \mathrm{m}$ image of the system (after Meyer et al. 2001; Smith et al. 2012).

source photon populations, with 1200 counts for the western source and 93 counts for the eastern source in the $0.3-2.0 \mathrm{keV}$ range, implying luminosities $L_{\mathrm{x}} \sim 2.0 \times 10^{29} \mathrm{erg} \mathrm{s}^{-1}$ for the western source (HD 113766B) and $L_{\mathrm{x}} \sim 1.6 \times 10^{28} \mathrm{erg} \mathrm{s}^{-1}$ for the eastern source (HD 113766A)

We show model fits to the spectrum of the brighter HD 113766B source (Figure 3(b)). Assuming solar metallicity (following $\mathrm{Fe} / \mathrm{H}=-0.01$ for $\mathrm{HD} 113766$ from Nordstrom et al. 2004), the spectrum can be fitted by an APEC emission model spectrum for collisionally ionized diffuse gas calculated using the ATOMDB code v2.0.1. The best-fit model appears to be very soft, with an APEC temperature of $0.50 \pm 0.06 \mathrm{keV}$ (or $6.7 \pm 0.8 \times 10^{6} \mathrm{~K}$ ), and a two-temperature model was not required to fit the data. While four to six times hotter than the effective coronal temperature of the $4.5 \mathrm{Gyr}$ old Sun $(\sim 0.10 \mathrm{keV})$, it is about the temperature found for other 10-20 Myr solar type stars of similar $L_{\mathrm{x}}$ (Suchkov et al. 2003; Telleschi et al. 2005). The shape of the spectrum, peaking at $\sim 0.8 \mathrm{keV}$, is also similar to the examples shown in Telleschi et al. (2005) in their X-ray spectral survey of young solar type stars. No strong emission lines above the background are obvious, but this is likely an effect of the coarse energy resolution of $\sim 50 \mathrm{eV}(1 \sigma)$ of the ACIS-S CCD coupled with the low number of total counts. The total detected flux from
$0.3-2.0 \mathrm{keV}$ is $1.4 \times 10^{-13} \mathrm{erg} \mathrm{cm}^{-2} \mathrm{~s}^{-1}$. The formal derived hydrogen upper limits of $N_{\mathrm{H}}<10^{20} \mathrm{~cm}^{-2}$ for the APEC models are consistent with the $\sim 5 \times 10^{19} \mathrm{~cm}^{-2}$ expected for a stellar source $\sim 123 \mathrm{pc}$ distant separated by an intervening interstellar $\mathrm{H}$ density of 0.1 particles $/ \mathrm{cm}^{3}$, suggesting that there is little in-system $\mathrm{H}$ absorption. A small hydrogen column is also consistent with there being little extinction toward the binary, as evidenced by comparing the observed $B-V$ for the system of $7.91-7.56=0.35$ to the predicted intrinsic $B-V=0.43$ (Pecaut et al. 2012).

With only 93 total counts, the total number of events obtained for the fainter HD 113766A "east" source is so small that we cannot used binned statistics for spectral fitting. Instead, we use a likelihood-based method (Cash statistic) which is appropriate for Poisson-distributed data. Forcing the $N_{\mathrm{H}}$ column to be the same as used in our HD $113766 \mathrm{~B}$ modeling, i.e., $N_{\mathrm{H}}<10^{20} \mathrm{~cm}^{-2}$, we find that the best fit is a stellar source with a very cool APEC temperature of $\sim 0.38 \mathrm{keV}$ or $5.6 \times 10^{6} \mathrm{~K}$, about $75 \%$ the temperature of the $\mathrm{B}$ source, and a total flux of $0.14 \times 10^{-13} \mathrm{erg} \mathrm{cm}^{-2} \mathrm{~s}^{-1}$, corresponding to an X-ray luminosity $L_{\mathrm{x}}=1.6 \times 10^{28} \mathrm{erg} \mathrm{s}^{-1}$ from $0.3-2.0 \mathrm{keV}$. As the low-count likelihood fitting method does not produce a formal $\chi^{2}$ statistic, we cannot derive formal confidence limits, but via forward modeling, Figure 3 


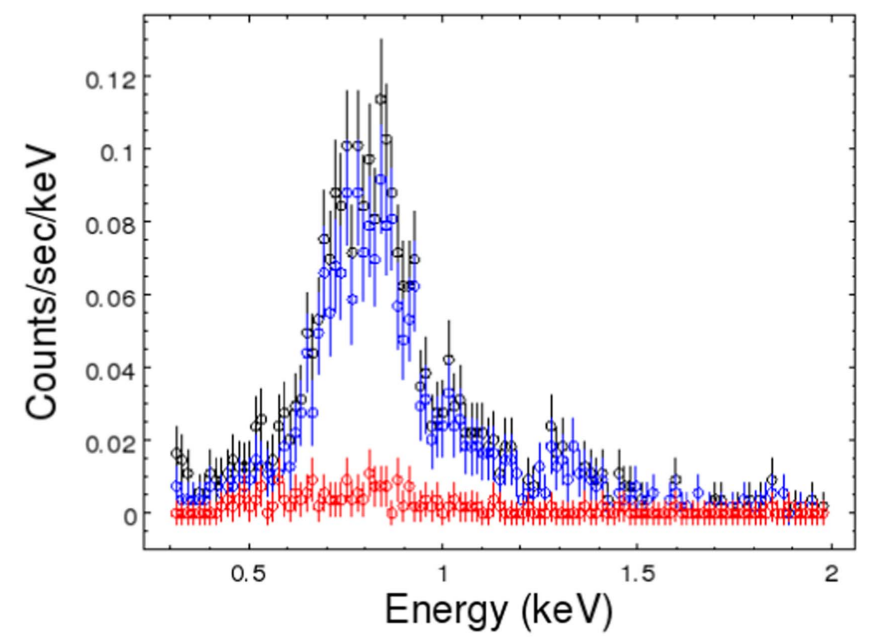

(a)

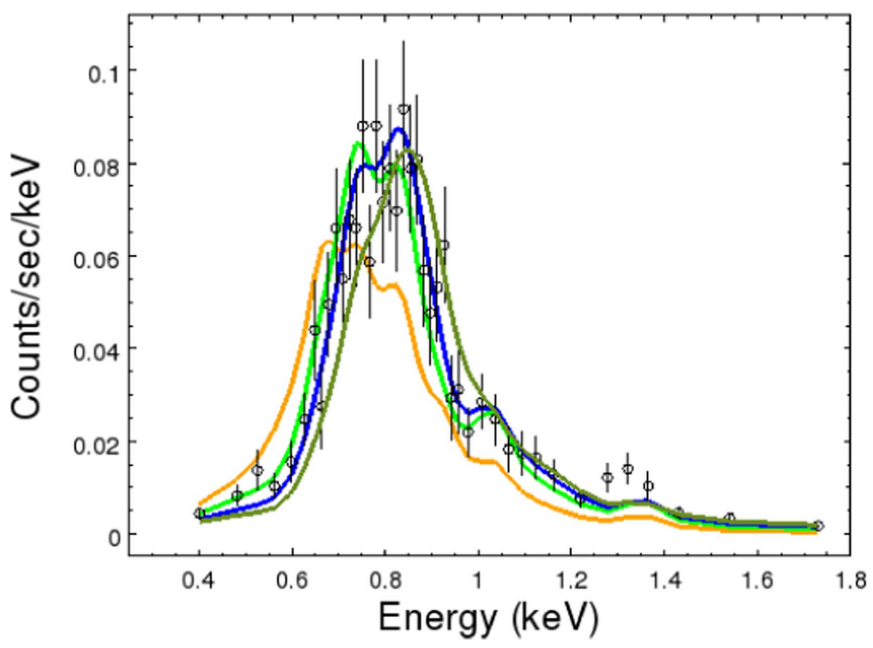

(b)

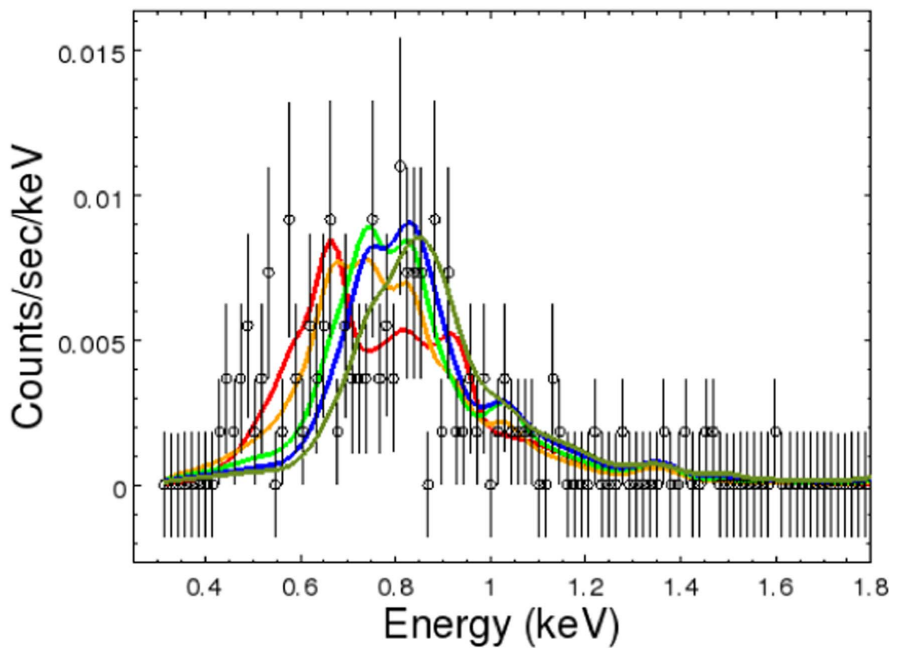

(c)

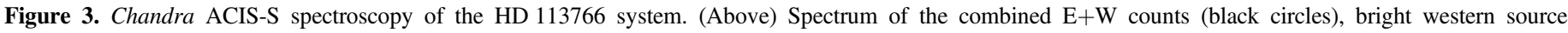

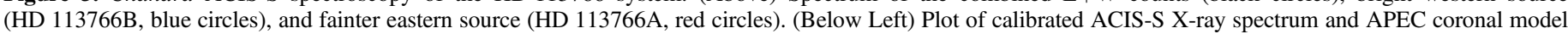

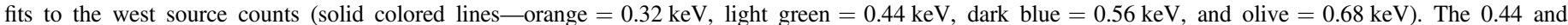

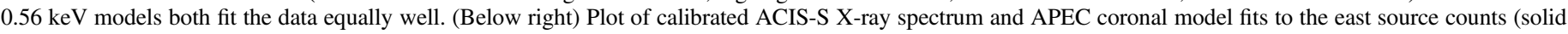

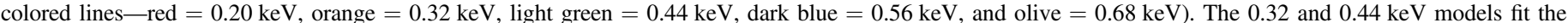
data the best.

shows that temperatures in the range $0.32-0.44 \mathrm{keV}$ (or $\left.(4.3-5.9) \times 10^{6} \mathrm{~K}\right)$ produce reasonable fits to the spectra.

\section{ANALYSIS}

In this section, we discuss the first-order implications of our Chandra observations of HD 113766 in comparison to X-ray observations of other stars. In the Discussion section, Section 4, we will relate their connections to the bigger picture of debris disk evolution.

\subsection{Luminosity Results}

Using Chandra, we have found an HD 113766 system with Chandra count rate and luminosity very close to the published RASS values. The observed X-ray emission can be accounted for by two stellar coronal sources located at the positions of HD 113766A and HD 113766B. HD 113766A is more than $12 \times$ fainter in the X-ray, consistent with the fact that it is the earlier of the two stars (Pecaut et al. 2012 classified
HD 113766A as F1-F3V, and Chen et al. 2011 have classified HD 113766B as F5V-F7V). The fact that HD 113766A is relatively X-ray faint at $\sim 2 \times 10^{28} \mathrm{erg} \mathrm{s}^{-1}$, at the lowest end of the Hipparcos-ROSAT survey luminosity range (Suchkov et al. 2003) is also consistent with it being a very early Fstar. This is in agreement with the comparison of our Chandra HD 113766A results to those found by Feigelson et al. (2006) for 51 Eri, a $23 \pm 3 \mathrm{Myr}$ (Bell et al. 2015) F0V star with $k T=0.2$ and $L_{\mathrm{x}}=1.4 \times 10^{28} \mathrm{erg} \mathrm{s}^{-1}$.

Similarly, the $L_{\mathrm{x}}=2 \times 10^{29} \mathrm{erg} \mathrm{s}^{-1}$ Chandra luminosity we find for HD 113766B is consistent with the $L_{\mathrm{x}} \sim$ $10^{29} \mathrm{erg} \mathrm{s}^{-1}$ luminosity reported for SAO 206462 by Müller et al. (2011), a young Herbig F8V star with a reported $3.9 \mathrm{hr}$ rotation period. An X-ray luminosity of $10^{29}-10^{30} \mathrm{erg} \mathrm{s}^{-1}$ is high for the average main-sequence star, but from the Kepler study of the rotation rates of stars (Meibom et al. 2011), as well as previous Hyades and Pleiades measurements (Stauffer et al. 1994; Stern et al. 1995; Güdel 2004), it is plausible for the very young HD 113766 F-stars to be brighter in the X-ray by 1-2 
orders of magnitude than their mature main-sequence F-star counterparts.

\subsection{Imaging Results}

Our Chandra data has, for the first time, resolved the two stars of HD 113766 in X-rays (Figure 2(a)). Examining in detail our deconvolved Chandra X-ray imagery (Figure 2(b)), we do not see any evidence for extended X-ray emission, or emission produced by anything but two point sources to the resolution limit of our mapping $(\sim 0$ !! 2 , or $25 \mathrm{AU}$ at $123 \mathrm{pc}$ distance).

The main unusual finding we have for this system is its brightness asymmetry. One of the main questions raised by this was the question of outbursts-i.e., was it possible that HD 113766B was flaring during the Chandra observations? Three lines of inquiry suggest that it was not: (a) the achieved count rate for the Chandra observations was within $10 \%$ of the rate estimated from the 1989 RASS counts, suggesting the system's X-ray luminosity had been stable over 21 years; (b) the time series of photons detected in our Chandra observations show no variability over and above a possible $\sim 2.8 \mathrm{hr}$, $\sim 20 \%$ peak-to-peak amplitude periodicity in the west source; and (c) the temperature of the HD 113766B X-ray spectrum is low compared to the typical 10-100 MK flare temperatures in stellar coronae (Feldman et al. 1995; Kashyap et al. 2002; Shibata \& Yokoyama 2002).

Given the incredibly dusty nature of the HD 113766A system and the lack of any dust signature in the HD 113766B system, coupled with the binary pair's coeval age of 10-16 Myr and the observed asymmetry in X-ray production, a natural inference from our results is that strong X-ray and stellar wind emission destroy and/or accelerate the removal of dust from a stellar system. Support for the latter finding comes from estimates of highly reduced dust lifetimes due to stellar wind drag (Chen et al. 2005, 2006, 2011), and by the anticorrelation between the lifetime of circumstellar dust and primary star X-ray luminosity found in TW Hya by Kastner et al. $(2004,2016)$. We will discuss this in more detail in Section 4.

\subsection{Spectral Results}

Spectrally, the observed HD 113766B X-ray emission is soft with $k T \sim 0.50 \mathrm{keV}$. This temperature is a factor of a few lower than a typical young G0 (c.f. Preibisch et al. 2005). This is not expected if the source of the flux is an $\alpha-\Omega$ dynamo, especially for a system in which there is evidence of rapid stellar rotation (Figure 1), unless the shear at the base of the convection zone is especially weak. Other possible effects on the observed spectrum, e.g., that a low coronal electron density or absorption by a large column of nearby $\mathrm{H}$ or absorption through the disk of circumstellar material would reduce the luminosity dramatically as well as selectively absorb lower-energy photons, is clearly not consistent with the observed ACIS-S spectrum for HD 113766B. The possibility that the soft spectrum can be attributed to accretion is remote, as (a) Chen et al. (2011) did not report any detectable $\mathrm{H}$-alpha emission in their Magellan/ MIKE $R \sim 50,000$ spectra for HD 113766, and (b) because the diskless star of the two in the binary is dominating the X-ray flux. Furthermore, $R \sim 10,000$ NIR spectroscopy of the HD $113766 \mathrm{~A}+\mathrm{B}$ system using the SPeX instrument at the NASA/IRTF $3 \mathrm{~m}$ in 2011 obtained by our group did not detect any $\mathrm{CO}$ or $\mathrm{HI}$ Brackett $\gamma$ line emission, which would be expected in the case of ongoing accretion (Connelly \& Greene 2010, 2014; Lisse et al. 2012, 2015). Related NIR measures of outflow activity in our SPeX spectrum, using Fe II, $\mathrm{He} \mathrm{I}$, and $\mathrm{H}_{2}$ lines (Connelly \& Greene 2014) are absent, arguing against outflow shocks as a soft X-ray source as well.

\section{DISCUSSION}

F-stars are intriguing objects in the X-ray. At the early end of their type, they are highly radiative and minimal X-ray emitters. At the late end of their type, they are highly convective and are strong X-ray emitters. Myr-old F-stars can be expected to be fast rotators, and more convective and X-ray active than their main-sequence cousins. The HD 113766 system has been alternately described as a $10-16 \mathrm{Myr}$ old F4/F6 or F3/F5 spectroscopic binary by most observers using optical photometry (Holden 1975, 1976; Houk 1978; Olsen \& Perry 1984; Hauck \& Mermilliod 1998; Mannings \& Barlow 1998; De Zeeuw et al. 1999; Fabricius \& Makarov 2000; Hoogerwerf et al. 2000; Madsen et al. 2002; Sartori et al. 2003; Hodge et al. 2004; Nordstrom et al. 2004; Chen et al. 2005; Rhee et al. 2008), and so we would have naively expected both stars to be rapidly rotating, highly convective, and X-ray bright. The fact that our east (A) source is $12 \times$ fainter in the X-ray is consistent with it being the earlier of the two stars. The fact that HD 113766A is X-ray faint in the absolute sense at $\sim 10^{28} \mathrm{erg} \mathrm{s}^{-1}$, at the lowest end of the Hipparcos-ROSAT survey (Suchkov et al. 2003), suggests that it is even earlier than $\mathrm{F} 3 \mathrm{~V}$ to $\mathrm{F} 4 \mathrm{~V}$, closer to $\mathrm{F} 2 \mathrm{~V}$, and the east component is a late, much more X-ray active F6V star, very much in agreement with Pecaut et al.'s (2012) recent optical spectroscopic reassessment of the system.

It also suggests that any stellar wind and high-energy stellar radiation effects on circumstellar material and objects are much more important in the HD 113766B system than the HD 113766A system, although the models of Ceisla \& Sandford (2012) argue that high-energy irradiation can drive important levels of organic synthesis in any water and organicrich circumstellar material present in either system-e.g., as has been reported for HD 113766A by Lisse et al. (2008). If local PPDs and the solar system's history are any guide, this irradiated material is likely to be astrobiologically important, as the larger pieces of it can be reincorporated into asteroids and planetesimals aggregating during the terrestrial planet-building era (which occurred from age 10-100 Myr in our system; Najita et al. 2010; Ercolano \& Glassgold 2013; Mendoza et al. 2013; Gudel 2015; Rosotti et al. 2015.). Many of these bodies will later accrete onto the terrestrial planets present in the system during the equivalent of our solar system's Late Veneer and Late Heavy Bombardment eras (Bottke et al. 2010; Raymond et al. 2013).

\subsection{Dust- $L_{x}$ Anticorrelation}

We have noted above the highly dusty nature of the X-ray faint HD 113766A system and the dust poor nature of the X-ray bright HD 113766B system. The X-ray observations of TW Hya stars by Kastner et al. $(2004,2016)$, and the fact that our own Sun is X-ray faint and planet rich versus the Kepler G-star average (Basri et al. 2010, 2011), suggests the distinct possibility of a causal connection between circumstellar dust excesses and low X-ray luminosities. Thus, it would seem that the asymmetric X-ray flux found for HD 113766A versus 
HD 113766 B by Chandra for the two coeval, similarly sized F-stars in this system requires a nature or nurture explanationdo dusty disks somehow diminish the observed (but corrected for dust absorption) X-ray flux (nature), or do disks thrive longer in low X-ray and stellar wind flux systems (nurture)?

To address the question of potential X-ray obscuration, it is important to consider the possibility that the circumstellar dust in HD $113766 \mathrm{~A}$ is strongly attenuating the primary's coronal emission via absorption and scattering. A simple calculation of $L_{\mathrm{IR}} / L_{\mathrm{bol}}$ (Lisse et al. 2008 and references therein) shows that while the HD 113766A circumstellar disk is very massive, $>1$ MMars, it intercepts and scatters at most $\sim 10^{-3}$ of the bolometric (mostly optical) flux of the star. Assuming normal X-ray scattering cross-sections (Morrison \& McCammon 1983) and a thin disk geometry, it is difficult to see how the HD 113766 A primary could be emitting roughly the same $L_{\mathrm{x}} \sim 2 \times 10^{29} \mathrm{erg} \mathrm{s}^{-1}$ as HD $113766 \mathrm{~B}$, only to have $90 \%$ of this flux absorbed by intervening circumstellar dust. Some enhancement of the observed extinction might be possible if the HD 113766A dust belts (Lisse et al. 2008) were in a fortuitous edge-on viewing situation, á la the Beta Pic system. However, IR imaging of the system does not argue for an unusual edge-on dust disk viewing geometry (Meyer et al. 2001), and the Spitzer IRS spectrum of the dust shows strong emission features, arguing against optically thick dust belts. Furthermore, the existence of soft $(<0.5 \mathrm{keV})$ X-rays from HD 113766A (Figure 3) indicates a very small amount of hydrogen gas along the line of sight $\left(<10^{20} / \mathrm{cm}^{2}\right)$ and thus a very small $A v<1$, even for gas to dust ratios as small as 25:1. Thus, dust obscuration of the HD 113766A X-rays is an unlikely reason for the observed X-ray asymmetry, and we again recover the reason for the star's X-ray luminosity asymmetry as being due to their differing stellar types.

On the other hand, previous authors have noted a possible dusty disk-X-ray luminosity anticorrelation due to the effects of a star's high-energy irradiation on material in orbit around it. Chandra observations of HD 98800, a quadruple system in the $10 \mathrm{Myr}$ old TW Hydrae association, have revealed that the $\mathrm{X}$-ray flux of the dusty binary system HD 98800B is $4 \times$ fainter than its dustless companion HD 98800A (Kastner et al. 2004). Ground-based searches for new 10 and $20 \mu \mathrm{m}$ excesses around proper-motion and X-ray selected K- and M-type members of the $10 \mathrm{Myr}$ old TW Hydrae association (Weinberger et al. 2004) and around proper-motion, X-ray, and lithiumselected F-, G-, K-, and M-type members of the $30 \mathrm{Myr}$ old Tucana-Horologium association (Mamajek et al. 2004) have been relatively unsuccessful. Recent work by Kastner et al. (2016) targeting the TW Hya M-star population has found an anticorrelation between a star's photospheric temperature and its Lx and the amount of IR excess flux arising from circumstellar material surrounding it.

Chen et al. (2005) summarized the possibilities when they wrote in examining their large sample of stars from the young, nearby Sco-Cen stars forming region: "Contrary to expectation, the infrared luminosity appears anti-correlated with X-ray luminosity, except for $30 \%$ of the objects that possess neither a ROSAT flux nor a MIPS $24 \mu \mathrm{m}$ excess. The anticorrelation can be naturally explained if stellar wind drag effectively removes dust grains around young stars with high X-ray coronal activity." Chen et al. (2011) reiterated this possible correlation, stating in the conclusions of their updated $\sim 400$ star Sco-Cen debris disk survey that they have found a "weak anticorrelation" between the ROSAT flux and the mid-IR flux from a system (the main problems with finding a stronger correlation were stated as the limiting sensitivities of the ROSAT survey and the derived stellar mass-loss rates).

\subsection{The Effects of Stellar Wind Drag}

Chen et al. $(2005,2006,2011)$ also noted the unusual nature of the HD 113766 system and its ROSAT detection, suggesting the important role that stellar wind drag could have in determining the lifetime of dust in a circumstellar debris disk versus infall onto the primary star. Specifically, they argued that the effects of a dense stellar wind on orbiting dust are similar to those of photons causing Poynting-Robertson drag, with the total contributions of the two mechanisms to the infall velocity $v_{\text {infall }}$ going as $v_{P-R} *\left(1+c^{2}[d M / d t]_{\text {wind }} / L_{*}\right)$. For the Sun, we have $[d M / d t]_{\text {wind,Sun }}=2.0 \times 10^{12} \mathrm{~g} \mathrm{~s}^{-1}$ and $L_{*}=3.9 \times 10^{33} \mathrm{erg} \mathrm{s}^{-1}$, implying $v_{\text {infall }} \sim 1.47 v_{P-R}$.

Photospheric X-ray emission is connected to stellar wind flow, as X-ray emission comes from the breaking of magnetic field lines, and the now open field lines guide energetic plasma away from the star, creating a stellar wind (Osten \& Wolk 2015). This connection allows us to estimate the stellar wind mass-loss rate from the stellar X-ray flux using $[d M / d t]_{\text {wind }}=C * 4 \pi R^{2} F_{\mathrm{x}, *}{ }^{1.3}$, where $C$ is a constant, $R_{*}$ is the stellar radius, and $F_{\mathrm{x}, *}$ is the $\mathrm{X}$-ray flux per unit stellar surface area (Wood et al. 2002, 2005, 2014). The constant C is determined by scaling from the solar result with $F_{\mathrm{x} \text {,Sun }}=3.7 \times 10^{4} \mathrm{erg} \mathrm{cm}^{-2} \mathrm{~s}^{-1}$ (Mamajek et al. 2002; Wood et al. 2002, 2005).

Assuming $\quad R_{\mathrm{HD} 113766 \mathrm{~A}} \sim R_{\mathrm{HD} 113766 \mathrm{~B}}=1.35 \quad R_{\mathrm{Sun}}$ (Lisse et al. 2008 and references therein), with our new Chandra results for the binary's X-ray fluxes we have $F_{\mathrm{x}, \mathrm{HD} 113766 \mathrm{~A}}=1.4 \times 10^{5} \mathrm{erg} \mathrm{cm}^{-2} \mathrm{~s}^{-1}$ and $F_{\mathrm{X}, \mathrm{HD}} 113766 \mathrm{~B}$ $=1.8 \times 10^{6} \mathrm{erg} \mathrm{cm}^{-2} \mathrm{~s}^{-1} \quad$ (compared to the $F_{\mathrm{x}, *}=9.2$ $\times 10^{5} \mathrm{erg} \mathrm{cm}^{-2} \mathrm{~s}^{-1}$ for both stars of the binary estimated by symmetrically assigning the RASS flux of the system; Chen et al. 2011). This implies $[d M / d t]_{\text {wind,HD } 113766 \mathrm{~A}}$ $=2.3 \times 10^{13} \mathrm{~g} \mathrm{~s}^{-1}$ and $[d M / d t]_{\text {wind,HD } 113766 \mathrm{~B}}=5.7$ $\times 10^{14} \mathrm{~g} \mathrm{~s}^{-1}$. With $L_{\mathrm{HD} 113766 \mathrm{~A}}=4.4 L_{\text {Sun }}$ for the $\mathrm{F} 2 \mathrm{~V}$ A-member (Lisse et al. 2008 and references therein; Chen et al. 2011), we find $v_{\text {infall }}=(2.2 \pm 0.24) v_{P-R}$ for HD 113766A. Assuming $L_{\mathrm{HD} 113766 \mathrm{~B}} \sim 2.3 L_{\mathrm{Sun}}$ for the F6V B-member (as the model ratio of F6V luminosity to F2V luminosity is $\sim 0.52)$, we have $v_{\text {infall }}=(58 \pm 3.3) v_{P-R}$ for HD 113766B.

We thus find, from our Chandra results, that the total drag effects for HD 113766B are $\sim 26$ times larger as for HD 113766A, and that stellar wind drag effects easily dominate the dynamical repulsive forces for HD 113766B. For HD 113766A, like our solar system, stellar wind drag effects are about equal in effect versus radiative drag forces. However, since $v_{P-R} \sim L_{*}$ (Burns et al. 1979), dust in the HD 113766A system still falls onto the primary about 6.6 times faster than in our solar system; but this is still rather slow compared to the 91 times faster than solar system infall rates experienced around HD 113766B.

In absolute terms, we have $t_{\text {infall }}=t_{\text {infall,solar-system }} *$ $\left(v_{\text {infall, solar-system }} / v_{\text {infall, } *}\right) \sim 400 * D^{2} /\left(0.2 / r_{\text {dust }}(\right.$ um $\left.)\right)$ years $*\left(v_{\text {infall,solar-system }} / v_{\text {infall, }}\right)$ (where $D=$ the distance from the dust particle to the central star and $r_{\text {dust }}$ is the dust particle radius; Burns et al. 1979). For the warm dust located at $\sim 1.8$ AU from the HD 133766A primary (Lisse et al. 2008), 
assuming it consists of a population of dust of which the largest and longest-lived grains initially present are the abundant $\sim 1 \mathrm{~mm}$ sized dust grains seen in solar system chondrules and comet trails common to the early solar system, this implies an infall time of $1.5 \times 10^{6}$ years for HD $113766 \mathrm{~A}$, or a disk clearing time in the order of $1 / 10$ th the primary's estimated total age. This suggests that the stability of a dense circumstellar dust disk, massing as least as much as Mars and created by currently ongoing intense asteroidal grinding or planetary accretion (Lisse et al. 2008; Olafsson et al. 2013), is quite plausible. Doing the same calculation for HD 113766B, we find a much shorter infall and disk clearing time of $\sim 0.9 \times 10^{5}$ years, less than $1 \%$ of the primary's estimated age, and it is not surprising that this system has quickly cleared out any dust created by collisions or impacts onto growing planetary embryos.

In summary, our Chandra HD 113766 binary X-ray results, taken together with those for the HD 98800 binary and other singleton debris disks (Kastner et al. 2004, 2016; Chen et al. 2005, 2011; Glauser et al. 2009), suggest that a requirement for circumstellar dust longevity is the lack of a strong primary stellar wind. If this is correct, then we can also expect that on the average young, early F-type stars should have a higher frequency of circumstellar dust disks than young, late-type Fstars as the X-ray luminosity and stellar wind activity increase across the class. It is also interesting to speculate that a relatively low X-ray and solar wind flux may have been the operative case in our early solar system-as suggested by the low activity rate for the Sun versus G-stars found in the Kepler sample (Basri et al. 2010, 2011).

\section{CONCLUSIONS}

We have used Chandra to obtain imaging spectroscopy of the close (1".4, or 170 AU separation), coeval (10-16 Myr old) F-star binary HD 113766 over 38 ks. All three Chandra lowenergy X-ray measures of this object-imaging, photometry, and spectroscopy-show a system with two detectable sources separated along an E-W line by $\sim 1$ !' 4, with the W source approximately 10 times as bright as the E source. The emission spectrum of each object is well fit by an APEC coronal emission model, although the emission appears to be rather soft for such young stars, $k T=0.30-0.50 \mathrm{keV}$, leading us to suspect that the coronal magnetic fields are weak in these F-stars. We find asymmetric X-ray emission from the two stellar sources, with the emission from the easternmost, the IR-extended primary object HD $113766 \mathrm{~A}$, only $\sim 10 \%$ that of the western star HD $113766 \mathrm{~B}$. There is no evidence for a $3 \mathrm{rd}$ member of the HD 113766 with mass greater than $0.1 M_{\text {Sun }}$ within $2^{\prime}$ of the $\mathrm{AB}$ pair. The X-ray emission from the HD $113766 \mathrm{~B}$ stronger source may vary with a $2.8 \pm 0.15 \mathrm{hr}$ period. For both stars, the strength of the X-ray emission varies inversely with the excess IR flux from circumstellar material. Stellar wind drag models corresponding to the $L_{\mathrm{x}} \sim 2 \times 10^{29} \mathrm{erg} \mathrm{s}^{-1}$ argue for a dust lifetime around HD $113766 \mathrm{~B}$ of $\sim 90,000$ years, suggesting that HD $113766 \mathrm{~B}$ efficiently clears any secondary dust out of its system, whereas HD 113766A, with $L_{\mathrm{x}} \sim 2 \times 10^{28} \mathrm{erg} \mathrm{s}^{-1}$ (12 times fainter than B) and dust lifetime $>1.5 \times 10^{6}$ years, could have created the dust seen today anytime within the last Myr. A similar situation has been found for a few other young debris disks, most notably HD 98800 by Kastner et al. (2004). Over the course of $1 \mathrm{Myr}$, the HD 113766A X-ray emission and stellar wind irradiation is high enough to drive important levels of organic synthesis in the orbiting circumstellar material, which is rich in water and carbonaceous materials (Lisse et al. 2008; Ceisla \& Sandford 2012).

The authors would like to thank J. Kastner, E. Mamajek, and J. Raymond for many useful discussions concerning X-ray emission from young stellar sources. C.M. Lisse gratefully acknowledges support for this work provided by the National Aeronautics and Space Administration through Chandra Award Number GO1-12028X issued by the Chandra X-ray Observatory Center (CXC). The CXC is operated by the Smithsonian Astrophysical Observatory for and on behalf of the National Aeronautics Space Administration under contract NAS8-03060.

\section{REFERENCES}

Backman, D., \& Paresce, F. 1993, in Protostars and Planets III (A93-42937 1790), ed. E. Levy, J. Lunine, \& M. Matthews (Tucson, AZ: Univ. Arizona Press), 1253

Basri, G., Walkowicz, L. M., Batalha, N., et al. 2011, AJ, 141, 20

Basri, G., Walkowicz, L. M., Batalha, N., et al. 2010, ApJL, 713, L155

Bell, C. P. M., Mamajek, E. E., \& Naylor, T. 2015, MNRAS, 454, 593

Bottke, W. F., Walker, R. J., Day, J. M. D., Nesvorny, D., \& Elkins-Tanton, L. 2010, Sci, 330, 1527

Burns, J. A., Lamy, P. L., \& Soter, S. 1979, Icar, 40, 1

Ceisla, F. J., \& Sandford, S. A. 2012, Sci, 336, 452

Chen, C. H., Jura, M., Gordon, K. D., \& Blaylock, M. 2005, ApJ, 623, 493

Chen, C. H., Sargent, B. A., Bohac, C., et al. 2006, ApJS, 166, 351

Chen, C. H., Mamajek, E. E., Bitner, M. A., et al. 2011, ApJ, 738, 122

Collins, K. A., Grady, C. A., Hamaguchi, K., et al. 2009, ApJ, 697, 557

Connelly, M. S., \& Greene, T. P. 2010, AJ, 140, 1214

Connelly, M. S., \& Greene, T. P. 2014, AJ, 147, 125

Currie, T., Balog, Z., Kenyon, S. J., et al. 2007, ApJ, 659, 599

Currie, T., Kenyon, S. J., Balog, Z., et al. 2008, ApJ, 672, 558

De Zeeuw, P. T., Hoogerwerf, R., de Bruijne, J. H. J., Brown, A. G. A., \& Blaauw, A. 1999, AJ, 117, 354

Drake, J. J., Braithwaite, J., Kashyap, V., Günther, H. M., \& Wright, N. J. 2014, ApJ, 786, 136

Draker, J. J., Laming, J. M., Widing, K. G., et al. 1995, Sci, 267, 1470

Ercolano, B., \& Glassgold, A. E. 2013, MNRAS, 436, 3446

Fabricius, C., \& Makarov, V. V. 2000, A\&A, 356, 141

Feigelson, E. D., Getman, K., Townsley, L., et al. 2005, ApJS, 160, 379

Feigelson, E. D., Lawson, W. A., Stark, M., Townsley, L., \& Garmire, G. P. 2006, AJ, 131, 1730

Feldman, U., Laming, J. M., \& Doschek, G. A. 1995, ApJ, 451, L79

Fruscione, A., McDowell, J. C., Allen, G. E., et al. 2006, Proc. SPIE, 6270, 60 Glauser, A. M., Güdel, M., Watson, D. M., et al. 2009, A\&A, 508, 247

Głebocki, R., \& Gnaciński, P. 2003, in The Catalogue of Rotational Velocities of Stars in Clusters, The Future of Cool-Star Astrophysics: 12th Cambridge Workshop on Cool Stars, Stellar Systems, and the Sun, ed. A. Brown, G. M. Harper, \& T. R. Ayres (Boulder, CO: Univ. Colorado), 823

Güdel, M. 2004, A\&ARv, 12, 71

Güdel, M. 2015, in EPJ Web of Conferences 102, Protoplanetary Disks: Theory and Modeling Meet Observations, ed. I. Kamp, P. Woitke, \& J. D. Ilee, 00015

Hauck, B., \& Mermilliod, M. 1998, A\&AS, 129, 431

Hodge, T. M., Kraemer, K. E., Price, S. D., \& Walker, H. J. 2004, ApJS, 151,299

Holden, F. 1975, PASP, 87, 945

Holden, F. 1976, PASP, 88, 52

Hoogerwerf, R. 2000, MNRAS, 313, 43

Houk, N. 1978, Michigan Spectral Survey (Ann Arbor: Dep. Astron., Univ. Michigan), 2

Kashyap, V. L., Drake, J. J., Gudel, M., \& Audard, M. 2002, ApJ, 580, 1118

Kastner, J., Huenemoerder, D. P., Schulz, N. S., et al. 2004, ApJL, 605, L49

Kastner, J., Principe, D. A., Punzi, K., et al. 2016, AJ, 152, 3

Lisse, C., Chen, C., Wyatt, M., \& Morlok, A. 2008, ApJ, 673, 1106

Lisse, C., Wyatt, M. C., Chen, C. H., et al. 2012, ApJ, 747, 93

Lisse, C., Sitko, M. L., Marengo, M., et al. 2015, ApJL, 815, L27

Madsen, S., Dravins, D., \& Lindegren, L. 2002, A\&A, 381, 446 
Maggio, A., Sanz-Forcada, J., \& Scelsi, L. 2011, A\&A, 527, A144

Mamajek, E. E., Meyer, M. R., Hinz, P. M., et al. 2004, ApJ, 612, 496

Mamajek, E. E., Meyer, M. R., \& Liebert, J. 2002, AJ, 124, 1670

Mannings, V., \& Barlow, M. J. 1998, ApJ, 497, 330

Meibom, S., Barnes, S. A., Latham, D. W., et al. 2011, ApJL, 733, L9

Mendoza, E., Almeida, G. C., Andrade, D. P. P., et al. 2013, MNRAS, 433,3440

Meyer, M. R., Backman, D., Mamajek, E. E., et al. 2001, BAAS, 33, 1420

Morrison, R., \& McCammon, D. 1983, ApJ, 270, 119

Müller, A., van den Ancker, M. E., Launhardt, R., et al. 2011, A\&A, 530, A85

Najita, J. R., Carr, J. S., Strom, S. E., et al. 2010, ApJ, 712, 274

Nordstrom, B., et al. 2004, A\&A, 418, 989

Olofsson, J., Juhász, A., Henning, Th., et al. 2012, A\&A, 542, A90

Olsen, E. H., \& Perry, C. L. 1984, A\&AS, 56, 229

Osten, R. A., \& Wolk, S. J. 2015, ApJ, 809, 79

Pecaut, M. J., Mamajek, E. E., \& Bubar, E. J. 2012, ApJ, 746, 154

Preibisch, T., Kim, Y-.C., Favata, F., et al. 2005, ApJS, 160, 401

Raassen, A. J. J., Mewe, R., Audard, M., et al. 2002, A\&A, 389, 228

Raymond, J. C., \& Smith, B. W. 1977, ApJS, 35, 419

Raymond, S. N., Schlichting, H. E., Hersant, F., \& Selsis, F. 2013, Icar, 226,671
Rhee, J. H., Song, I., \& Zuckerman, B. 2008, ApJ, 675, 777

Rosotti, G. P., Ercolano, B., \& Owen, J. E. 2015, MNRAS, 454, 2173

Sartori, M. J., Lepine, J. R. D., \& Dias, W. S. 2003, A\&A, 404, 913

Shibata, K., \& Yokoyama, T. 2002, ApJ, 577, 422

Smith, R., Wyatt, M. C., \& Haniff, C. A. 2012, MNRAS, 422, 2560

Stauffer, J. R., Caillault, J.-P., Gagne, M., Prosser, C. F., \& Hartmann, L. W. 1994, ApJS, 91, 625

Stern, R. A., Schmitt, J. H. M. M., \& Kahabka, P. T. 1995, ApJ, 448, 683

Suchkov, A. A., Makarov, V. V., \& Voges, W. 2003, ApJ, 595, 1206

Telleschi, A., Güdel, M., Briggs, K., et al. 2005, ApJ, 622, 653

Van Leeuwen, F. 2007, A\&A, 474, 653

Voges, W., Aschenbach, B., Boller, Th., et al. 1999, A\&A, 349, 389

Weinberger, A. J., Becklin, E. E., Zuckerman, B., \& Song, I. 2004, AJ, 127, 2246-51

Wood, B. E., \& Laming, M. J. 2013, ApJ, 768, 122

Wood, B. E., Müller, H.-R., Redfield, S., \& Edelman, E. 2014, ApJL, 781, L33

Wood, B. E., Müller, H.-R., Zank, G. P., \& Linsky, J. L. 2002, ApJ, 574,412

Wood, B. E., Müller, H.-R., Zank, G. P., Linsky, J. L., \& Redfield, S. 2005, ApJL, 628, L143 